\title{
Auszug aus dem Protokoll der
} Generalversammlung in Davos 25.9.2015

Beginn 12:34 Uhr, anwesend : 30 Mitglieder

1. Begrüßung und Feststellung der Beschlussfähigkeit

A. Klauser begrüßt die Teilnehmer/Innen. Statutengemäß wird die Generalversammlung nach einigen Minuten nochmals eröffnet. Die Beschlussfähigkeit kann somit festgestellt werden.

2. Genehmigung des Protokolls der Generalversammlung vom 30.10.2014 Das Protokoll wird einstimmig angenommen.

3. Bericht der Präsidentin

A. Klauser stellt die neuen Kursleiter S. Meng (Kopf/Hals) und C. Dejaco (Bewegungsapparat) vor, die bei der letzten Vorstandssitzung der ÖGUM anerkannt wurden.

Im Mai 2015 haben sich in Salzburg die Arbeitsgruppenleiter und -stellvertreter zur jährlichen erweiterten Vorstandssitzung getroffen. Dieses Treffen diente neben dem allgemeinen internen Informationsaustauch auch dazu, um die Zertifizierungs- richtlinien zu aktualisieren bzw. auf diese den Schwesterngesellschaften anzupassen.

In der Vorstandssitzung am 24. September wurde beschlossen, den Arbeitskreisen einmal jährlich bis zu $€ 500$,- für Sitzungen (Saalmiete, Verpflegung) zur Verfügung stellen.

A. Klauser stellt das Konzept der Studentenförderung vor, welches im 2.-3. Ausbildungsjahr beginnt und auf ÖGUM, Dreiländertreffen und Universität basiert. Förderung von vorerst 5 Studenten pro Jahr über 3 Jahre, beginnend mit US2016 in Leipzig:

- Gratis Teilnahme am Dreiländertreffen

- Bezahlung eines Anwenderseminares

- Im Rahmen des Dreiländertreffens: Vorstellung eigener Ziele bei der ÖGUM Vorstandssitzung

- Praktische Erfahrungen in der Sonohöhle als Hands-On-Modell

- Voraussetzung: Wahlfach Ultraschall an der Universität ist verpflichtend
- Förderung eines ÖGUM-Ultraschallkurses bis $€ 150$,-

- Abschlussgespräch bzw. -prüfung bei ÖGUM-Vorstandssitzung mit Diplomverleihung

„ÖGUM Studententutor“

Diesbezüglich wird noch ein Curriculum erarbeitet und beim nächsten Treffen der Vorstände im Mai 2016 den Schwesterngesellschaften vorgestellt.

4. Bericht des Kassiers

C. Kollmann stellt den Finanzbericht des Jahres 2014 vor. Der Mitgliederstand beträgt derzeit 940 .

C. Brezinka erkundigt sich, ob es möglich wäre, eigene Veranstaltungen finanziell über die ÖGUM zu organisieren. A. Klauser antwortet, dass sie als Präsidentin der Gesellschaft in diesem Fall die gesamte finanzielle Verantwortung übernimmt, wie es beispielsweise bei den Dreiländertreffen der Fall ist.

Die Anfrage wird einem Juristen vorgelegt, um die Möglichkeiten zu eruieren und eine Lösung zu finden um das Kurswesen in Österreich unterstützen zu können.

5. Entlastung des Vorstandes ÖGUM-Rechnungsprüfer T. Ybinger bestätigt die korrekte Finanzgebarung des Vereins nach dem österr. Vereinsgesetz für den Prüfungszeitraum 01.01.2014-31.12.2014, sowie die ordnungsgemäße Verwendung der Vereinsmittel laut den Vereinsstatuten. Die Belege wurden stichprobenartig geprüft.

G. Mathis stellt den Antrag auf Entlastung des Vorstands, welcher einstimmig angenommen wird. A. Klauser bedankt sich im Namen des Vorstandes für das entgegengebrachte Vertrauen.

6. Berichte der Landesgruppen Folgende Berichte wurden vorgetragen:

Landesgruppe Wien: Am 12.12.2014 fand im Rahmen einer interdisziplinären Fortbildungsveranstaltung die Jahreshauptversammlung mit Neuwahlen statt.

Landesgruppe Vorarlberg: Kurse wurden veranstaltet, mit insgesamt 250 Teilnehmer/Innen. 
Landesgruppe Burgenland: Neuwahlen, mit KRAGES/Barmherzige Brüder wurden Kurse organisiert.

Landesgruppe Niederösterreich: Kurse wurden organisiert, Sitzung mit Neuwahlen veranstaltet Landesgruppe Salzburg: Kurse wurden veranstaltet, Neuwahlen stehen an.

Landesgruppe Tirol: Kurse wurden veranstaltet (MSK, Gefäße, Abdomen).

7. Thieme Zeitschrift „Ultraschall in der Medizin“

H. Kathrein erklärt wie es letztes Jahr zu der Vertragskündigung der Gesellschaften mit Thieme gekommen ist. Die DEGUM war damals die treibende Kraft und schlug bei dem Treffen der Vorstände in Seefeld letztes Jahr eine taktische Kündigung vor, um bessere Vertragsbedingungen zu erhalten. Nach dieser Kündigung hat es vielversprechende und kooperative Verhandlungen mit Thieme gegeben. Nun liegt ein neuer Vertragsentwurf vor, über den die Mitglieder jetzt abstimmen müssen.

H. Kathrein stellt den Vertrag kurz vor und schildert die Eckpunkte:
Die Mitglieder der ÖGUM können als Bezugsform der Zeitschrift die Printund Online-Ausgabe oder die OnlineOnly-Ausgabe wählen. Erklärt sich ein Mitglied hierzu nicht ausdrücklich, wird der Bezug der Zeitschrift als Print- und Online-Ausgabe fortgeführt.

Der Verlag wird die bereits entwickelte Lese-App für die Zeitschrift, die speziell auf Tablet-PCs ausgerichtet ist, weiterentwickeln. Die App wird den ordentlichen Mitgliedern kostenfrei zur Verfügung gestellt.

Die Abstracts zum Dreiländertreffen erscheinen zukünftig ausschließlich als Online-Sonderausgabe der Zeitschrift und werden über den OnlineAuftritt der Zeitschrift allen Mitgliedern und darüber hinaus auch NichtMitgliedern frei zugänglich gemacht.

In den nächsten Monaten werden die Mitglieder gefragt, ob eine OnlineOnly oder eine Print- und OnlineAusgabe gewünscht wird. Bei der Online-Only-Version wird der Mitgliedsbeitrag um die Druck- und Versandkosten der Zeitschrift reduziert.
Der Vertrag wird zur Wahl gestellt und einstimmig angenommen.

\section{Allfälliges}

W. Eppel weist darauf hin, dass Stufeninhaber der DEGUM, die älter als 65 Jahre sind, keine Rezertifizierungen mehr durchführen müssen. Er fragt, wie das bei der ÖGUM gehandhabt wird. Der Vorstand hat diesbezüglich noch keine Information erhalten und wird das mit den Schwesterngesellschaften besprechen.

Ende: 13:37 Uhr

Das vollständige Protokoll finden unsere Mitglieder im Memberbereich vor.

Allen unseren Mitgliedern wünschen der Vorstand und die Geschäftsstelle einen gesunden und erfolgreichen Ausklang des Jahres und bedanken uns für die konstruktive Zusammenarbeit während des zu Ende gehenden Jahres! 